\title{
Índice de alimentación saludable e indicador sodio/(potasio+calcio) en estudiantes universitarios chilenos
}

\author{
Healthy eating index and sodium/(potassium+calcium) indicator in Chilean university students
}

Eduard Maury-Sintjago ${ }^{*}$ orcid.org/0000-0002-9482-8268

Alejandra Rodríguez-Fernández ${ }^{1}$ orcid.org/0000-0002-3982-3165

Julio Parra-Flores ${ }^{1}$ orcid.org/0000-0002-4835-9747

1. Universidad del Bío-Bío, Facultad de Ciencias de la Salud y de los Alimentos, Departamento de Nutrición y Salud Pública. Chillán,
Chile

\section{Resumen}

Introducción: Los hábitos alimentarios poco saludables de los estudiantes universitarios constituyen una preocupación importante para la atención medica-nutricional futura. Objetivo: Determinar el Índice de Alimentación Saludable (IAS) e indicador sodio/(potasio+calcio) en universitarios chilenos. Materiales y métodos: Investigación analítica, de corte transversal con una muestra no probabilística de 420 estudiantes, a quienes se aplicó encuesta de información sociodemográfica y antropométrica. Con el cuestionario de frecuencia de consumo y recordatorio de 24 horas se determinó el IAS e indicador sodio/(potasio+calcio). Se aplicó estadística descriptiva e inferencial utilizando el programa Statistical Package for the Social Sciences v22.0. Resultados: El peso, talla e Índice de Masa Corporal, presentaron diferencias estadísticamente significativas, según sexo $(p<0,05)$. Misma situación fue encontrada en el promedio de ingesta de calorías, lípidos, carbohidratos, fibra, calcio y sodio $(p<0,05)$. La ingesta de frutas, verduras y lácteos bajos en grasa fue mayor en mujeres $(p<0,01)$. La mayoría de los sujetos presentaron alimentación poco saludable, siendo mayor en el caso de los hombres $(p<0,05)$. Sólo el $6 \%$ de los hombres y $17 \%$ de las mujeres tenían una dieta cardio-protectora. Conclusiones: Los estudiantes presentan una dieta con deficiente ingesta de micronutrientes cardioprotectores, hecho que incrementa el riesgo de desarrollar enfermedades cardiovasculares.

Palabras clave: Estudiantes; alimentación saludable; ingesta dietética; enfermedad cardiovascular. (Fuente: DeCS, Bireme).

\begin{abstract}
Introduction: Unhealthy eating habits of university students constitutes an important concern for future medical and nutritional care. Objective: To determine the Healthy Eating Index (HEI) and the sodium/(potassium+calcium) indicator in Chilean university students. Materials and methods: An analytical cross-sectional research was carried out with a non-probabilistic sample of 420 students, who answered a survey covering sociodemographic and anthropometric issues. The HEI and sodium/(potassium+calcium) indicator were determined using the consumption frequency and 24-hour reminder questionnaire. A descriptive and inferential statistical analysis was applied through the SPSS (v22.0) program. Results: The weight, height and the body mass index (BMI) of the participants showed statistically significant differences according to their gender $(\mathrm{p}<0.05)$. A similar pattern was found in the average intake of calories, lipids, carbohydrates, fiber, calcium and sodium $(\mathrm{p}<0.05)$. Intake of fruits, vegetables and low-fat dairy products was higher in women $(\mathrm{p}<0.01)$. The majority of participating students had an unhealthy diet, with the situation being more severe in men ( $\mathrm{p}<0.05)$. Only $6 \%$ of men and $17 \%$ of women had a cardio-protective diet. Conclusions: Students follow diets with a deficient intake of cardio-protective micronutrients, which increases the risk of developing cardiovascular diseases.
\end{abstract}

Key words: Students; healthy eating; dietary intake; cardiovascular disease. (Source: DeCS, Bireme).

\footnotetext{
*Autor de correspondencia

Eduard Maury Sintjago

e-mail: emaury@ubiobio.cl
} 


\section{Introducción}

Las enfermedades no transmisibles se han posicionado como las principales causas de morbimortalidad a nivel mundial, asociadas a factores de riesgo como el estrés, factores genéticos, nivel de actividad física, ingesta dietética, estado nutricional, entre otras ${ }^{(1,2) .}$

Los gobiernos han realizado numerosos esfuerzos para revertir esta situación y promover estrategias en la población que favorezcan estilos de vida saludables. Destacan las guías alimentarias basadas en alimentos (GABA) $)^{(3,4)}$ e instrumentos gráficos propios de cada país como la pirámide alimentaria, ovalo nutricional, plato saludable, olla alimentaria, entre otras. Las guías alimentarias independiente del país son consideradas como un aporte esencial para el logro de las metas establecidas por la Organización Mundial de la Salud (OMS) sobre alimentación, actividad física y salud, convirtiéndose además en herramientas locales para fomentar hábitos alimentarios saludables y para medir la calidad de la dieta a nivel poblacional(5).

Otros indicadores que permiten evaluar la calidad dietaria de grupos poblacionales son el índice de alimentación saludable y el indicador sodio/(potasio+calcio)(6). El índice de alimentación saludable permite evaluar el nivel de cumplimiento que tiene un grupo poblacional en relación a las guías alimentarias y recomendaciones de su país(7). Mientras que la relación sodio/(potasio+calcio) a partir de la evaluación de ingesta dietética evalúa nutrientes claves que participan en la patogénesis de enfermedades cardiovasculares, como la hipertensión arterial(6-8). Lo anterior es particularmente importante en edades tempranas de la vida, como en la primera infancia, adolescencia y adulto joven. Por ello, la intervención en estos grupos de población es fundamental para prevenir en edades más tardías las consecuencias, secuelas o complicaciones ocasionadas por las enfermedades crónicas de origen nutricional.

Estudios manifiestan que los estudiantes universitarios son un grupo de riesgo al no presentar un estado nutricional óptimo, asociado a los escasos conocimientos de alimentación saludable y a la influencia de las empresas de marketing publicitarios; además se ha descrito que los estudiantes universitarios (quienes en su mayoría son adultos jóvenes 17-25 años), presentan riesgo de malnutrición por exceso con deficiencia de micronutrientes debido a los hábitos alimentarios( $(9)$. Algunas de las posibles causas son abandonar su núcleo familiar para trasladarse a los recintos universitarios, lo que les obliga a hacerse responsable de su alimentación, sumado a la dinámica académica (horarios irregulares), la oferta de alimentos poco equilibrados, el consumo de bebidas azucaradas y/o estimulantes, consumo de café, supresión del desayuno y/o almuerzo, baja disponibilidad de recursos económicos, entre otros $(7,9)$.

Khawar en su estudio con estudiantes universitarios canadienses evidenció que, en promedio, el 19\% de los estudiantes estaban por debajo del extremo inferior de AMDR (sigla asociada a la expresión inglesa de Rango de distribución aceptable de macronutrientes) para carbohidratos y el $16 \%$ estaban por encima del extremo superior de AMDR para la grasa total(10). Por otro lado, Muñoz-Cano et al., muestran que el $80,2 \%$ de los estudiantes mexicanos que participaron en el estudio, presentaron un índice de alimentación en la categoría "poco saludable"(11). Finalmente, Espinoza y Hernández, mostraron que los estudiantes universitarios peruanos presentaban un alto consumo de sal corriente y sodio(12).

Por lo anterior, el objetivo del presente estudio fue determinar el índice de alimentación saludable e indicador sodio/(potasio+calcio) en estudiantes universitarios chilenos. El propósito es aportar evidencia sobre la calidad dietética de los estudiantes y de este modo contribuir al establecimiento de políticas de promoción de salud y alimentación saludable para este grupo específico.

\section{Materiales y métodos}

\section{Tipo de estudio}

Se utilizó una investigación analítica de corte transversal.

\section{Población y muestra}

La muestra del estudio estuvo constituida por 420 estudiantes, de ambos sexos (69\% mujeres y 31\% hombres) que aceptaron participar voluntariamente del estudio. Se trata de una muestra no probabilística. El mayor porcentaje (95\%) de los estudiantes tenía una edad entre 20 y 30 años. Como criterios de inclusión los sujetos participantes debían ser estudiantes universitarios regulares, 
mayores de edad, no consumir suplementos dietéticos ni medicamentes y ser aparentemente sanos.

\section{Características bio-sociodemográficas}

A los participantes se les aplicó un instrumento que recogía información bio-sociodemográfica como edad, peso, talla, comuna de procedencia, semestres cursados, carrera de estudios y hábitos durante la alimentación. Las mediciones antropométricas (peso $\mathrm{y}$ talla) se realizaron según procedimientos estandarizados y utilizando los valores de referencia(13).

\section{Frecuencia de consumo y recordatorio de 24 horas (24H)}

Para estimar la frecuencia de consumo alimentario se solicitó al participante que indicara la frecuencia (diario, semanal, quincenal o mensual) del consumo de distintos alimentos (organizados por grupos con nutrientes comunes), se registró la cantidad consumida por vez y a partir de éste se calculó la cantidad de energía y nutrientes consumidos por día. Por otro lado, se aplicó un recordatorio de 24 horas, para este método los sujetos de estudio debieron enumerar los alimentos y las porciones que consumieron en las últimas 24 horas (promedio de lo consumido en dos días de la semana más un día del fin de semana), los cuales fueron transformados en gramos a partir del cual se calculó su composición nutricional. Para determinar el aporte calórico y nutricional de cada alimento o preparación se utilizó la tabla de composición de alimentos y preparaciones chilenas( ${ }^{(14) .}$

\section{Índice de Alimentación Saludable}

Para determinar el IAS se utilizó la metodología propuesta por Pinheiro y Atalah, validada para la población chilena(7). A partir de la información recogida en el instrumento de frecuencia de consumo alimentario, se procedió a calcular y analizar el número de porciones consumidas al día por grupo de alimentos: cereales, frutas, verduras, lácteos (altos, medios y bajos en grasa), carnes y pescados (altas y bajos en grasas), pescados, legumbres, aceites y azucares. El IAS incluye 10 variables con un máximo de 10 puntos cada uno (mínimo 0 y máximo 100), se utilizó el siguiente rango de clasificación de la calidad de la alimentación: $\geq 80$ puntos "Saludable"; 51-79 puntos "Necesita cambios", $\leq 50$ puntos "Poco saludable".

\section{Indicador sodio/(potasio+calcio)}

A partir del cálculo de la ingesta de nutrientes se determinó el coeficiente sodio/(potasio+calcio), según la metodología propuesta y validada por Durán y Labraña(6). El coeficiente sodio/(potasio+calcio) es un indicador dietario cuyo valor se determina a partir de las recomendaciones de ingesta diaria para los minerales según sexo y grupo etario. En la población universitaria cuyo promedio de edad oscila entre los 20 y 30 años, se espera que el cociente sea $<0,46$ para una dieta con bajo riesgo cardiovascular, debido a que lo recomendado es sodio (2300mg), calcio (1200mg) y potasio (3800mg).

\section{Análisis Estadístico}

Se verificó la distribución normal de los resultados con la prueba de Shapiro-Wilks. Se empleó la prueba de $t$-student para comparar las diferencias de medias entre hombres y mujeres. Todos los análisis fueron ejecutados con el programa SPSS (Statiscal Package for the Social Sciences), versión 22.0. En todos los casos se utilizó un nivel de significancia $\alpha<0,05$.

\section{Consideraciones Éticas}

El comité de ética de la Facultad de Salud y Ciencias de la Actividad Física de la Universidad SEK revisó y aprobó el estudio. A los participantes se les invitó a participar de forma voluntaria y todos firmaron un consentimiento informado. Los procedimientos empleados estuvieron de acuerdo con las normas éticas de la declaración de Helsinki y el Consejo de Organizaciones Internacionales de las Ciencias Médicas (CIOMS)(15).

\section{Resultados}

\section{Características generales y de ingesta en la muestra de estudio}

En la tabla 1, se muestran las características generales e ingesta de macronutrientes y electrolitos de los sujetos de estudios, según sexo. Se puede observar que hombres y mujeres tenían edades similares $(p>0,05)$. El peso, la talla y el Índice de Masa Corporal (IMC) presentaron diferencias estadísticamente significativas $(p<0,05)$. Misma situación fue encontrada en el promedio de ingesta de calorías, lípidos, Carbohidratos (CHO), fibra, calcio y sodio $(p<0,05)$. No resultaron diferentes estadísticamente la ingesta de proteínas y potasio $(p>0,05)$. 


\section{Ingesta de porciones por grupo de alimentos en universitarios, según sexo}

La tabla 2 muestra que el consumo promedio de porciones por alimento sólo fue estadísticamente diferente entre mujeres y hombres respecto a la ingesta de frutas, verduras, lácteos bajo en grasa, siendo mayor en mujeres $(p<0,01)$.

\section{Índice de alimentación saludable e indicador sodio/potasio-calcio}

Respecto al índice de alimentación saludable, tanto en mujeres como hombres la clasificación con mayor porcentaje fue de "poco saludable" con $84 \%$ y $91 \%$, respectivamente. El 16\% de las mujeres y $9 \%$ de hombres clasifican en la categoría de "necesita cambios". Las mujeres presentaron menores porcentaje de dieta poco saludable que los hombres $(p=0,015)$.

En relación al indicador sodio/(potasio+calcio), se encontró que el $94 \%$ de los hombres y el $83 \%$ de las mujeres tuvieron el indicador por sobre el 0,46, lo que indica que la mayoría de los sujetos no tienen una dieta cardio-protectora. El promedio de indicador para las mujeres fue de 0,76 y de 1,29 para hombres, ambos presentan un riesgo elevado (tabla 3).

Tabla 1. Características generales y de ingesta de macronutrientes y electrolitos en los sujetos de investigación, según sexo

\begin{tabular}{|c|c|c|c|}
\hline Variables & $\begin{array}{c}\text { Mujer } \\
(n=290)\end{array}$ & $\begin{array}{l}\text { Hombre } \\
(n=130)\end{array}$ & $p$ \\
\hline Edad (años) & $24,78 \pm 4,50$ & $24,35 \pm 1,95$ & 0,632 \\
\hline Peso (kg) & $61,19 \pm 9,58$ & $73,61 \pm 6,88$ & 0,001 \\
\hline Talla (m) & $1,61 \pm 0,005$ & $1,69 \pm 0,005$ & 0,001 \\
\hline $\operatorname{IMC}\left(\mathrm{kg} / \mathrm{m}^{2}\right)$ & $23,44 \pm 3,37$ & $25,79 \pm 2,67$ & 0,002 \\
\hline Energía (Kcal) & $1645,00 \pm 547,58$ & $\begin{array}{r}2419,88 \pm \\
769,12\end{array}$ & 0,001 \\
\hline Proteínas (g) & $69,52 \pm 59,12$ & $92,46 \pm 37,65$ & 0,067 \\
\hline Lípidos (g) & $53,72 \pm 33,93$ & $72,65 \pm 43,18$ & 0,024 \\
\hline $\begin{array}{l}\text { Carbohidratos } \\
\text { (g) }\end{array}$ & $215,16 \pm 108,18$ & $\begin{array}{r}384,21 \pm \\
134,69\end{array}$ & 0,001 \\
\hline Fibra (g) & $14,96 \pm 11,28$ & $23,76 \pm 12,45$ & 0,001 \\
\hline Calcio (mg) & $555,340 \pm 330,78$ & $\begin{array}{r}447,26 \pm \\
296,01\end{array}$ & 0,001 \\
\hline Sodio (mg) & $1528,30 \pm 801,80$ & $\begin{array}{r}2629,57 \pm \\
1362,15\end{array}$ & 0,001 \\
\hline Potasio (mg) & $1433,01 \pm 636,59$ & $\begin{array}{r}1658,93 \pm \\
732,30 \\
\end{array}$ & 0,135 \\
\hline
\end{tabular}

Datos expresados en promedio \pm desviación estándar.

Prueba $t$-student. Significancia estadística $p<0,05$
Tabla 2. Ingesta por grupos de alimentos (porciones) en los sujetos de estudio, según sexo

\begin{tabular}{|c|c|c|c|c|}
\hline \multicolumn{2}{|c|}{ Grupo de alimento } & $\begin{array}{c}\text { Mujer } \\
(n=290)\end{array}$ & $\begin{array}{l}\text { Hombre } \\
(n=130)\end{array}$ & $p$ \\
\hline \multicolumn{2}{|l|}{ Cereales } & $4,48 \pm 2,95$ & $4,54 \pm 3,30$ & 0,891 \\
\hline \multicolumn{2}{|l|}{ Frutas } & $1,78 \pm 1,63$ & $0,91 \pm 1,16$ & 0,001 \\
\hline \multicolumn{2}{|l|}{ Verduras } & $2,24 \pm 1,75$ & $1,50 \pm 1,48$ & 0,030 \\
\hline \multirow{3}{*}{ Lácteos* } & Altos & $0,80 \pm 1,08$ & $0,74 \pm 0,69$ & 0,666 \\
\hline & Medios & $0,74 \pm 0,75$ & $0,65 \pm 0,76$ & 0,437 \\
\hline & Bajos & $0,71 \pm 0,90$ & $0,30 \pm 0,69$ & 0,001 \\
\hline \multirow{2}{*}{ Carnes* } & Altas & $1,03 \pm 1,18$ & $1,11 \pm 1,01$ & 0,626 \\
\hline & Bajas & $0,88 \pm 0,98$ & $0,65 \pm 0,76$ & 0,095 \\
\hline \multicolumn{2}{|l|}{ Pescados } & $0,85 \pm 1,36$ & $0,50 \pm 0,74$ & 0,056 \\
\hline \multicolumn{2}{|l|}{ Legumbres } & $0,97 \pm 0,91$ & $0,51 \pm 0,79$ & 0,061 \\
\hline \multicolumn{2}{|l|}{ Aceites } & $1,08 \pm 1,10$ & $1,21 \pm 1,11$ & 0,447 \\
\hline \multicolumn{2}{|l|}{ Azucares } & $0,97 \pm 1,62$ & $1,29 \pm 1,83$ & 0,205 \\
\hline
\end{tabular}

Tabla 3. Índice de alimentación saludable e indicador sodio/(potasio+calcio) en los sujetos de estudio, según sexo

\begin{tabular}{lccc}
\hline & $\begin{array}{c}\text { Mujer } \\
\text { (n=29) }\end{array}$ & $\begin{array}{c}\text { Hombre } \\
(\mathbf{n = 1 3 0 )}\end{array}$ & $\boldsymbol{p}$ \\
\hline \multicolumn{2}{l}{ Índice De Alimentación Saludable } \\
Poco saludable & 84,0 & & \\
Necesita cambios & 16,0 & 91,0 & \\
Saludable & -- & -- & 0,015 \\
Indicador Sodio/(Potasio + Calcio) & & \\
$<0,46$ & 17,0 & 6,0 & \\
$\geq 0,46$ & 83,0 & 94,0 & 0,030 \\
\hline
\end{tabular}

Datos expresados en porcentajes.

Prueba $\mathrm{Chi}^{2}$. Significancia estadística $p<0,05$

\section{Discusión}

La importancia que representa una alimentación adecuada en todo el ciclo vital es inobjetable. Por ello, prevenir el desarrollo de enfermedades no transmisibles mediante una ingesta dietética saludable es la principal estrategia de disminución del gasto público y baja productividad ocasionada por el aumento del reposo médico asociado a esta morbilidad(16,17). Por tal motivo, la detección de hábitos alimentarios inadecuados y su modificación a hábitos más saludables es una de las acciones que deben ser incorporadas en la formulación de políticas de promoción de la salud.

Nuestro estudio encontró que el $69 \%$ de los sujetos era eutrófico y el 31\% tenía malnutrición por exceso (datos no mostrados). El porcentaje de sujetos con sobrepeso y obesidad son superiores a los 
reportados por Duran et al., en estudiantes chilenos $(29,6 \%)^{(18)}$, a los de Muñoz et al., en estudiantes españoles $(13,4 \%)^{(19)}$ y de Khan et al., en estudiantes malayos $(6,4 \%)^{(20)}$.

Por otro lado, se encontró una diferencia estadísticamente significativa en el consumo de calorías, carbohidratos, fibra, calcio y sodio según sexo. En todos los casos el consumo fue superior en el sexo masculino. Estos hallazgos concuerdan con los reportados por De Piero et al., con población argentina, dónde indican que existen diferencias por sexo, siendo el consumo más elevado en hombres comparado con mujeres(21). En ese mismo estudio, se mostró un consumo energético de $3091 \pm 1252$ kcal (hombres) y $2330 \pm 945 \mathrm{kcal}$ (en mujeres), valores muy superiores a los reportados en nuestro estudio (1645 kcal mujeres y $2419,8 \mathrm{kcal}$ hombres). Vázquez et al., en otro estudio en Argentina muestra valores muy similares a los nuestros tanto en mujeres $(1563,0 \pm 338,5)$ como en hombres $(2316,8 \pm 273,5)^{(22)}$.

Para la determinación del índice de alimentación saludable fue necesario realizar el cálculo de ingesta de porciones por grupo de alimentos. En nuestro estudio la mayoría de los hombres (91\%) y de las mujeres (84\%) tuvieron un índice de alimentación saludable categorizado en "poco saludable", seguido por "necesita cambios" (9\% hombres y $16 \%$ mujeres) y ningún sujeto se ubicó en "saludable". Los resultados de este estudio son similares a los reportados por Muñoz-Cano et al., en su estudio con estudiantes mexicanos dónde reporta que la categoría "poco saludable" representó el 80,2\% de la muestra, seguido por un 19,7\% "necesita cambios" y $0,1 \%$ "saludable"(11). Por su parte, Cervera-Burriel, reporta en su estudio con españoles universitarios que ninguno se ubicó en la categoría de "saludable"(23).

No existen estudios que evalúen el indicador sodio/potasio-calcio en estudiantes universitarios. Sin embargo, Sumalla-Cano et al., en su estudio con estudiantes del Cono Sur muestra un consumo promedio de sodio, calcio y potasio de $1225,74 \mathrm{mg}$, $740,0 \mathrm{mg}$ y $1548,46 \mathrm{mg}$, respectivamente ${ }^{(24)}$. Estos hallazgos difieren levemente de nuestro estudio, donde el consumo de sodio diferenciado por sexo es el que más varía. Por su parte, De Piero et al., en estudiantes argentinos muestra una ingesta de sodio más cercano a los reportados en este estudio
$(646,8 \pm 348,7 \mathrm{mg})(21)$. Crovetto et al., en su estudio con 97 estudiantes universitarios muestra que más del $57 \%$ de la población tienen un consumo de moderado a alto de sodio(25). El consumo de potasio se encontró muy bajo, se sabe que el adecuado consumo de potasio está asociado con menor riesgo de padecer hipertensión(26).

\section{Conclusiones}

La calidad de la ingesta alimentaria de la muestra de estudio evidencia la necesidad de establecer programas de promoción de la salud y compromiso desde las instituciones educativas para favorecer un mejor estado de salud y hábitos saludables de vida. La mayoría de los estudiantes evaluados presentaron una pobre calidad dietaria con una ingesta deficiente de micronutrientes cardioprotectores que incrementa el riesgo de enfermedades cardiovasculares. No obstante, lo anterior, se hace necesario incrementar el número de casos a estudiar y realizar un muestreo probabilístico para superar las limitaciones del presente estudio.

Conflicto de intereses: Los autores declaran que no existen conflictos de interés.

\section{Referencias}

1. GBD 2015 Risk Factors Collaborators. Global, regional, and national comparative risk assessment of 79 behavioral, environmental and occupational, and metabolic risks or clusters of risks, 1990-2015: a systematic analysis for the Global Burden of Disease Study 2015. Lancet. 2016;388(10053):1659-1724. https://doi.org/10.1016/S0140-6736(16)31679-8

2. Baird J, Jacob Ch, Barker M, Fall M, Hanson M, Harvey N, et al. Developmental Origins of Health and Disease: A Lifecourse Approach to the Prevention of NonCommunicable Diseases. Healthcare. 2017;14(5):2-12. https://doi.org/10.3390/healthcare5010014

3. Wijesinha-Bettoni R, Khosravi A, Sherman J, HernándezGarbanzo Y, Vargas M, Ramos AI. Implementing food-based dietary guidelines for policies, programmes and nutrition education. UNSCN News. 2017;42:77-89. Disponible en: https://www.unscn.org/uploads/web/news/UNSCNNews42-2017.pdf

4. OMS. Global Nutrition Policy Review 2016-2017, Country progress in creating enabling policy environments for promoting healthy diets and nutrition. 2018. Disponible en https://www.who.int/publications/i/item/978924151487 3

5. FAO. Second International Conference on Nutrition. Framework for action. Rome, Nov 2014. Disponible en: http://www.fao.org/3/a-ml542e.pdf 
6. Durán E, Labraña A. Indicadores de Calidad de la Dieta. Editorial Universidad de Concepción. 1a Edición. Concepción 2019.

7. Pinheiro A, Atalah E. Propuesta de una metodología de análisis de la calidad global de la alimentación. Rev Med Chile. 2005;133:175-182. http://dx.doi.org/10.4067/S0034-98872005000200004

8. Mirmiran P, Gaeini Z, Bahadoran Z, Ghasemi A, Reza $\mathrm{N}$, Tohidi M, Azizi F. Urinary sodium to potassium ratio: $\mathrm{A}$ simple and useful indicator of diet quality in populationbased studies. Nutrition \& Dietetics. 2019. https://doi.org/10.21203/rs.2.12332/v1

9. Peltzer K, Pengpid S, Samuels T, Keser N, Mantilla C, Rahamefy 0, et al. Prevalence of overwerigth/obesity and its associated factors among university students from 22 Countries. Int J Environ Res Public Health. 2014;11(7):7425-7441. https://doi.org/10.3390/ijerph110707425.

10. Khawar H. Energy and nutrient intake among university students enrolled in an introductory nutrition course. 2018. A Thesis submitted to The Faculty of Graduate Studies of The University of Manitoba in partial fulfillment of the requirements of the degree of Master of Science. The University of Manitoba Canadá. Disponible en: https://mspace.lib.umanitoba.ca/bitstream/handle/1993/ 33623/khawar_hibah.pdf?sequence=1\&isAllowed=y

11. Muñoz-Cano J, Cordova-Hernández J, Valle-Leveaga D. El índice de alimentación saludable de estudiantes de nuevo ingreso a una universidad de México. Nutr Hosp. 2015;31(4):1582-1588.

http://dx.doi.org/10.3305/nh.2015.31.4.8401

12. Espinoza L, Hernández E. Determinación del consumo de sal corriente y sodio en estudiantes universitarios de la Facultad de Medicina. Universidad Nacional Mayor de San Marcos. 2016. Tesis para optar al grado académico de Magíster en Ciencias de los Alimentos. Universidad Nacional de San Marcos, Lima-Perú. Disponible en: https://hdl.handle.net/20.500.12672/4808

13. Barrera G. Evaluación nutricional del crecimiento y del riesgo cardiovascular y metabólico. 13va edición. Santiago de Chile: Editorial Universidad de Chile; 2018.

14. Zacarías I, Barrios L, González CG, Loeff T, Vera G. Tabla de Composición de Alimentos. INTA. Santiago de Chile: Editorial Universidad de Chile. 2018.

15. Consejo de Organizaciones Internacionales de las Ciencias Médicas /Organización Mundial de la Salud, CIOMS/OMS. 2002. Pautas éticas internacionales para la investigación biomédica en seres humanos. [Documento Electrónico]. Disponible en: http://www.ub.es/rceue/archivos/Pautas_Eticas_Internac. pdf.

16. Bloom DE, Cafiero ET, Jané-Llopis E, Abrahams-Gessel S, Bloom LR, Fathima S, et al. The Global Economic Burden of Noncommunicable Diseases. Geneva: World Economic Forum. 2012. PGDA Working Paper No. 87. Disponible en: https://www.world-heart-federation.org/wpcontent/uploads/2017/05/WEF_Harvard_HE_GlobalEcono micBurdenNonCommunicableDiseases_2011.pdf

17. Chaker L, Falla A, Van der Lee S, Muka T, Imo D, Jaspers L, et al. The global impact of non-communicable diseases on macro-economic productivity: a systematic review. European Journal of Epidemiology. 2015;30:357. https://doi.org/10.1007/s10654-015-0026-5.
18. Duran S, Vasquez A, Morales G, Schifferli I, Sanhueza C, Encina C, et al. Consumo de estevia en estudiantes universitarios chilenos y su asociación con el estado nutricional. Nutr Hosp. 2015;32(1):362-366. http://dx.doi.org/10.3305/nh.2015.32.1.8961

19. Muñoz G, Lozano E, Romero M, Santiago C, Pérez D, Veiga P. Evaluación del consumo de alimentos de una población de estudiantes universitarios y su relación con el perfil académico. Nutr Hosp. 2017;34(1):134-143. http://dx.doi.org/10.20960/nh.989.

20. Khan M, Draman S, Khan A, Usman M. "Comparison of nutritional status of university students of two Asian countries". Nutrition \& Food Science, 2012;42(5):332-338. https://doi.org/10.1108/00346651211266845

21. De Piero A, Bassett N, Rossi A, Sammán N. Tendencia en el consumo de alimentos de estudiantes universitarios. Nutr Hosp. 2015;31(4):1824-1831. http://dx.doi.org/10.3305/nh.2015.31.4.8361

22. Vázquez $M$, Witriw $A$, Reyes C. Estudio preliminar sobre la ingesta alimentaria en estudiantes universitarios de las carreras de medicina y arquitectura de la Universidad de Buenos Aires. Diaeta. 2010:28(131):14-17. Disponible en: http://www.scielo.org.ar/scielo.php?script=sci_arttext\&pid $=$ S1852-73372010000200003\&lng=es\&nrm=iso\&tlng=es

23. Cervera-Burriel Faustino. Hábitos alimentarios en estudiantes universitarios: Universidad de Castilla-La Mancha. Estudio Piloto en la Universidad Virtual de Túnez. 2014. Tesis Doctoral. Disponible en: https://ruidera.uclm.es/xmlui/bitstream/handle/10578/5 953/TESIS\%20Cervera\%20Burriel.pdf?sequence=1

24. Sumalla-Cano IE, Domínguez I, Calderón R, García A, Fernández F, Gracia S, et al. Valoración del perfil e ingesta de nutrientes de un grupo de estudiantes iberoamericanos de postgrados en nutrición. Nutr Hosp. 2013;28(2):532540. http://dx.doi.org/10.3305/nh.2013.28.2.6242

25. Crovetto M, Figueroa B, González L, Jeria A, Ramírez N. Guías alimentarias y su cumplimiento en estudiantes universitarias, Valparaíso, 2013, Chile. Rev Chil Nutr. 2015;42(2):164-172. http://dx.doi.org/10.4067/S071775182015000200008

26. OMS. Biblioteca electrónica de documentación científica sobre medidas nutricionales (eLENA). Abril 2019. Disponible en: https://www.who.int/elena/titles/potassium_cvd_adults/e s/ 